\title{
Tunable metal-insulator transition in double-layer graphene heterostructures
}

\author{
L. A. Ponomarenko ${ }^{1}$, A. K. Geim ${ }^{1,2}$, A. A. Zhukov², R. Jalil' ${ }^{2}$, S. V. Morozov ${ }^{1,3}$, K. S. Novoselov ${ }^{1}$, \\ I. V. Grigorieva' , E. H. Hill ${ }^{2}$, V. V. Cheianov ${ }^{4}$, V. I. Fal'ko ${ }^{4}$, K. Watanabe ${ }^{5}$, T. Taniguchi ${ }^{5}$ \\ and R. V. Gorbachev ${ }^{2 \star}$
}

\begin{abstract}
Disordered conductors with resistivity above the resistance quantum $h / e^{2}$ should exhibit an insulating behaviour at low temperatures, a universal phenomenon known as a strong (Anderson) localization ${ }^{1-3}$. Observed in a multitude of materials, including damaged graphene and its disordered chemical derivatives ${ }^{4-10}$, Anderson localization has not been seen in generic graphene, despite its resistivity near the neutrality point reaching $\approx h / e^{2}$ per carrier type ${ }^{4,5}$. It has remained a puzzle why graphene is such an exception. Here we report a strong localization and the corresponding metal-insulator transition in ultra-high-quality graphene. The transition is controlled externally, by changing the carrier density in another graphene layer placed at a distance of several $\mathrm{nm}$ and decoupled electrically. The entire behaviour is explained by electron-hole puddles that disallow localization in standard devices but can be screened out in doublelayer graphene. The localization that occurs with decreasing rather than increasing disorder is a unique occurrence, and the reported double-layer heterostructures presents a new experimental system that invites further studies.
\end{abstract}

Resistivity values $\approx h / e^{2}$ indicate that the electron mean free path $l$ is shorter than the Fermi wavelength $\lambda_{\mathrm{F}}$, so that quantum interference becomes a dominant feature in electron diffusion, leading to Anderson localization in the absence of phase-breaking processes at low temperatures $(T)$. The scope of this phenomenon extends beyond electronic systems-into optical and acoustic phenomena as well ${ }^{1-3}$ - but not generic graphene, which remains metallic at liquid-helium $T$ (refs 4,5) and exhibits only a weak $T$ dependence that can be explained by phonons and thermally excited carriers $^{11}$. Earlier theoretical studies have suggested that Dirac electrons can evade localization for certain types of disorder ${ }^{3,12-15}$, with the extreme example being graphene subjected to a smooth Coulomb potential ${ }^{16,17}$. However, for generic disorder that involves scattering between the two graphene valleys, the localization is expected to be unavoidable ${ }^{3,18,19}$. Experiments do not show this.

In this Letter, we describe a double-layer electronic system made of two closely-spaced but electrically isolated graphene monolayers sandwiched in boron nitride. In the following, the two layers in the double layer graphene (DLG) heterostructure are referred to as the studied and control layers. At low doping $n_{\mathrm{C}}$ in the control layer, the studied layer exhibits the standard behaviour with a minimum metallic conductivity of $\sim 4 e^{2} / h$. However, for $n_{\mathrm{C}}>10^{11} \mathrm{~cm}^{-2}$, the resistivity $\rho$ of the studied layer diverges near the neutrality point (NP) at $T<70 \mathrm{~K}$. This divergence can be suppressed by a small perpendicular field $B<0.1 \mathrm{~T}$, which indicates that this is an interference effect rather than a gap opening. We attribute the metal-insulator transition (MIT) to the recovery of an intrinsic behaviour such that graphene exhibits Anderson localization if its $\rho$ reaches values of $\approx h / e^{2}$ per carrier type. Normally, this intrinsic MIT is obscured by charge inhomogeneity in the form of electronhole puddles ${ }^{20-24}$. Within each puddle, graphene is sufficiently away from the NP and remains metallic. Then, resistivity of the percolating electron-hole system with leaking $\mathrm{p}-\mathrm{n}$ boundaries ${ }^{16,17}$ assumes a value of $\sim h / e^{2}$ with little $T$ dependence (conceptually this value has little in common with the similar value required for Anderson localization $)^{23,24}$. The control layer can screen out the fluctuating background potential and suppress electron-hole puddles, revealing the intrinsic properties at the NP. This reconciles the metallic behaviour normally observed in graphene with the localization expected for large $\rho$ and supports the idea that the minimum conductivity that tends to assume values close to $4 e^{2} / h$ is due to electron-hole puddles ${ }^{23,24}$.

The studied devices were fabricated by sandwiching two graphene monolayers with thin hexagonal-BN crystals. In a multistep procedure, described in the Supplementary Information, a graphene monolayer was transferred onto a $20-30 \mathrm{~nm}$ thick BN crystal that was first prepared on top of an oxidized Si wafer. Then, the graphene was covered with another BN crystal (spacer), which was followed by transfer of the second graphene layer. Both layers were shaped into multiterminal devices aligned above each other and having separate electrical contacts (Fig. 1a). Individual steps were similar to those described in refs 25,26 but the whole fabrication process involved three dry transfers and alignments, four nonconsecutive rounds of electron-beam lithography, three rounds of plasma etching and two separate metal depositions. The resulting DLG heterostructures are schematically shown in Fig. 1a (for images, see Supplementary Information). We made several such devices with channel widths of $1-2 \mu \mathrm{m}$. They exhibited $\mu$ of $30-120 \times 10^{3} \mathrm{~cm}^{2} \mathrm{~V}^{-1} \mathrm{~s}^{-1}$ and little chemical doping. The bottom layer encapsulated in $\mathrm{BN}$ always had higher $\mu$ and changed little after exposure to air $^{26}$ whereas the quality of the top layer gradually decayed. For this particular study, we employed three multiterminal devices with sufficiently thick BN spacers to avoid any detectable tunnel current between graphene layers $(<0.1 \mathrm{nA})$. The spacers had thicknesses $d \approx 4,12$ and $16 \mathrm{~nm}$. All the devices exhibited a similar MIT behaviour, although the insulating state was much more pronounced for devices with smaller $d$ and higher $\mu$, as described below.

\footnotetext{
${ }^{1}$ School of Physics and Astronomy, University of Manchester, Manchester M13 9PL, UK, ${ }^{2}$ Manchester Centre for Mesoscience and Nanotechnology, Manchester M13 9PL, UK, ${ }^{3}$ Institute for Microelectronics Technology, 142432 Chernogolovka, Russia, ${ }^{4}$ Physics Department, University of Lancaster, Lancaster LA1 4YB, UK, ${ }^{5}$ National Institute for Materials Science, 1-1 Namiki, Tsukuba, 305-0044, Japan. *e-mail: blizza@gmail.com.
} 


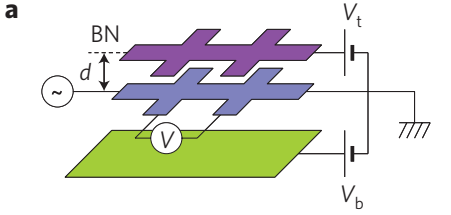

c



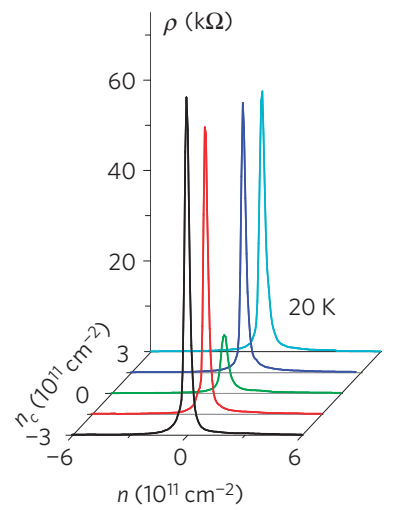

Figure 1 | Electron transport in graphene-BN heterostructures. a, Schematic view of our heterostructure devices and measurement geometry. $\mathbf{b}, \mathbf{c}, \rho$ as a function of $n$ in the studied graphene layer for different doping $n_{C}$ of the control layer at two temperatures. The device has a $4 \mathrm{~nm}$ BN spacer.

With reference to Fig. 1a, we employed the following scheme of measurements. A voltage $V_{\mathrm{t}}$ was applied between the graphene layers, and this electrically doped both of them with carriers of the opposite sign. The bottom layer could also be gated by a voltage $V_{\mathrm{b}}$ applied to the Si wafer. Because of the low density of states, graphene can provide only a partial screening and, therefore, $V_{\mathrm{b}}$ induced carriers in the top layer as well. This influence was weaker than on the bottom layer and depended on $n$ in the latter. By measuring the Hall resistivity $\rho_{x y}$ we could determine $n$ in each of the layers (Supplementary Information). We usually fixed $V_{\mathrm{t}}$ to define a nearly constant $n$ in the top layer and swept $V_{\mathrm{b}}$ to vary $n$ in the bottom layer. Normally, we studied the higher- $\mu$ bottom layer and used the top layer as control. In this configuration, the insulating state reached higher $\rho$. If the studied and control layers were swapped, the behaviour remained qualitatively the same (Supplementary Information) but lower $\mu$ resulted in lower $\rho$ of the insulating state.

Our main result is illustrated by Fig. 1, which shows two sets of standard curves $\rho(n)$ for the studied layer at different $n_{\mathrm{C}}$. At $70 \mathrm{~K}$, the control layer has little effect on the studied layer, and all the curves in Fig. 1b look no different from those observed in the standard devices ${ }^{4}$ or for graphene on BN (GBN; ref. 25). However, at low $T$ and for high doping of the control layer $\left(n_{\mathrm{C}}>10^{11} \mathrm{~cm}^{-2}\right)$, graphene exhibits a radically different behaviour (Fig. 1c). In this regime, $\rho$ at the NP acquires a strong $T$ dependence and easily overshoots the threshold value of $h / e^{2}$. To elucidate this observation, Fig. 2 shows further examples of $\rho(n, T)$ for high and low doping of the control layer. In the case of large $n_{\mathrm{C}}$ (Fig. 2a), $\rho$ exhibits an insulating $T$ dependence. In contrast, zero $n_{\mathrm{C}}$ results in a much weaker $T$ dependence that can be explained by thermally excited carriers (Fig. 2b). Outside a relatively narrow interval $|n| \leq 10^{10} \mathrm{~cm}^{-2}$ and above $70 \mathrm{~K}$, the behaviour of graphene was practically independent of $n_{\mathrm{C}}$.

The $T$ dependence of the maximum resistivity at the NP, $\rho^{\mathrm{NP}}$, is shown in more detail in the inset of Fig. $2 b$ for zero and high doping of the control layer. The insulating state is more pronounced for $d=4 \mathrm{~nm}$ but remains clear also for the $12 \mathrm{~nm}$ device (note the logarithmic scale). For $d=4 \mathrm{~nm}$ and below $4 \mathrm{~K}, \rho^{\mathrm{NP}}$ could reach into the $\mathrm{M} \Omega$ range (Supplementary Information), an increase by $2-3$ orders of magnitude with respect to the standard behaviour. The high- $\rho$ regime is found to be difficult to probe because of a strong nonlinearity caused by a crosstalk between the measurement current and $V_{\mathrm{t}}$, the effect specific to DLG devices with small $d$ (see Supplementary Information). To assure the linear response in this regime, we had to measure $I-V$ curves at every gate voltage and, to avoid these difficulties, we limited our studies mostly to $T>4 \mathrm{~K}$ and $\rho^{\mathrm{NP}}<100 \mathrm{k} \Omega$.
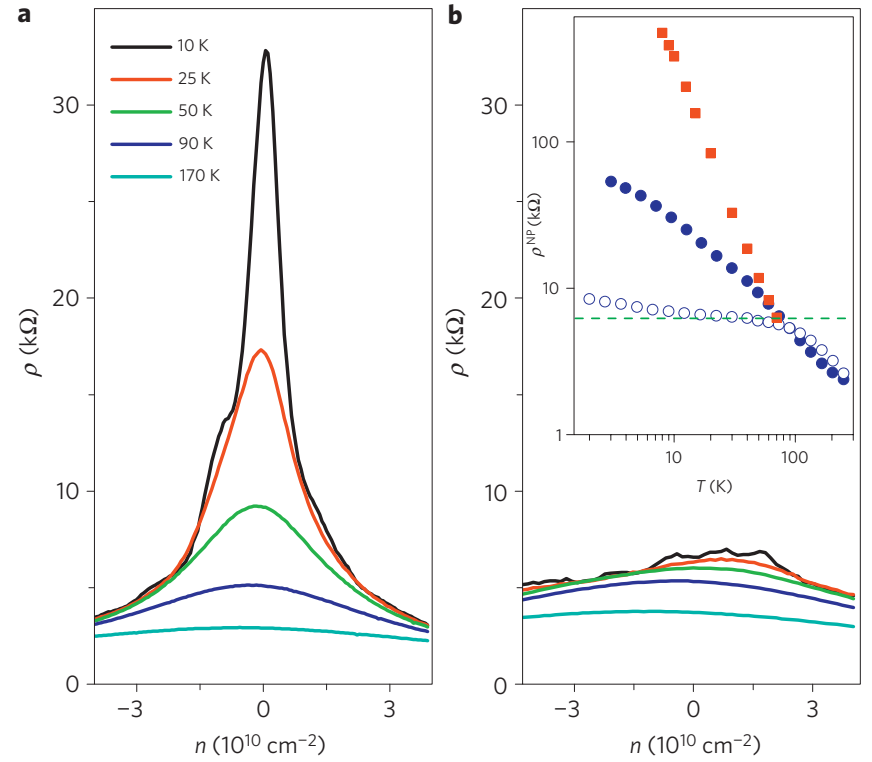

Figure 2 | Resistivity of the studied layer at different $T$ for high and low doping of the control layer. $\mathbf{a}, \mathbf{b}$, Correspond to $n_{C} \approx 3 \times 10^{11} \mathrm{~cm}^{-2}$ and zero $n_{C}$, respectively. Here, we have chosen to plot data for $d \approx 12 \mathrm{~nm}$. For our thinnest spacer $(\approx 4 \mathrm{~nm}), \rho^{\mathrm{NP}}$ becomes very large at low $T$ (inset) and continuous curves $\rho(n)$ are difficult to measure because of crosstalk nonlinearities (Supplementary Information). The inset shows the $T$ dependence of $\rho^{\mathrm{NP}}$ for the device in the main figure at both $n_{\mathrm{C}}$ (open and filled circles) and for the $4 \mathrm{~nm}$ device at $n_{C} \approx 5 \times 10^{11} \mathrm{~cm}^{-2}$ (squares). The dashed line indicates the threshold value for Anderson localization, $\rho=h / 4 e^{2}$.

The influence of the adjacent layer immediately invites one to consider interlayer Coulomb interactions. Indeed, the relevant energy scale is $e^{2} / \varepsilon d \sim 50 \mathrm{meV}$, that is, the interactions may be a significant factor $(\varepsilon \approx 5$ is BN's dielectric constant). For example, one can imagine that the interactions open an excitonic-like gap at the Dirac point. We have ruled out this possibility by magnetic field measurements. In the gapped case, $B$ is expected to enhance the confinement and, hence, the binding energy. In contrast, our devices exhibit a pronounced negative magnetoresistance in non-quantizing $B$ (Fig. 3). The insulating behaviour is suppressed in characteristic $B^{*} \approx 10 \mathrm{mT}$ (Fig. 3), well below the onset of Landau quantization. Figure 3 also shows that the MIT is again confined to $|n| \leq 1 \times 10^{10} \mathrm{~cm}^{-2}$.

Another interesting observation is that the insulating state has always developed at $\rho>h / 4 e^{2}$ (Figs 1-3). This is seen most clearly in the inset of Fig. 2, where the curves depart from each other above the dashed line marking $h / 4 e^{2}$. In the insulating state, $\rho^{\mathrm{NP}}$ is found to follow a power-law dependence $1 / T^{v}$, where $v$ varied from sample to sample, reaching a value close to two in the device with $d \approx 4 \mathrm{~nm}$. The characteristic $T$ at which the insulating state started to develop can be attributed to the fact that above $70 \mathrm{~K}$ the concentration of excited carriers at the NP exceeded $\approx 10^{10} \mathrm{~cm}^{-2}$, beyond which no MIT could be observed even at low $T$.

The suppression of the MIT by non-quantizing $B$ is a clear indication that localization plays an important role, such that $B$ breaks down the time-reversal symmetry and destroys the interference pattern that developed due to self-intersecting trajectories ${ }^{1-3}$. The strong localization scenario is also consistent with the onset of the insulating state at $\rho^{\mathrm{NP}} \approx h / 4 e^{2}$, which corresponds to the resistivity quantum per carrier type. However, localization in graphene cannot possibly be explained without intervalley scattering ${ }^{3,18,19}$. A tempting line of argument would be to invoke charge fluctuations in the control layer to explain its influence on the studied layer. However, 


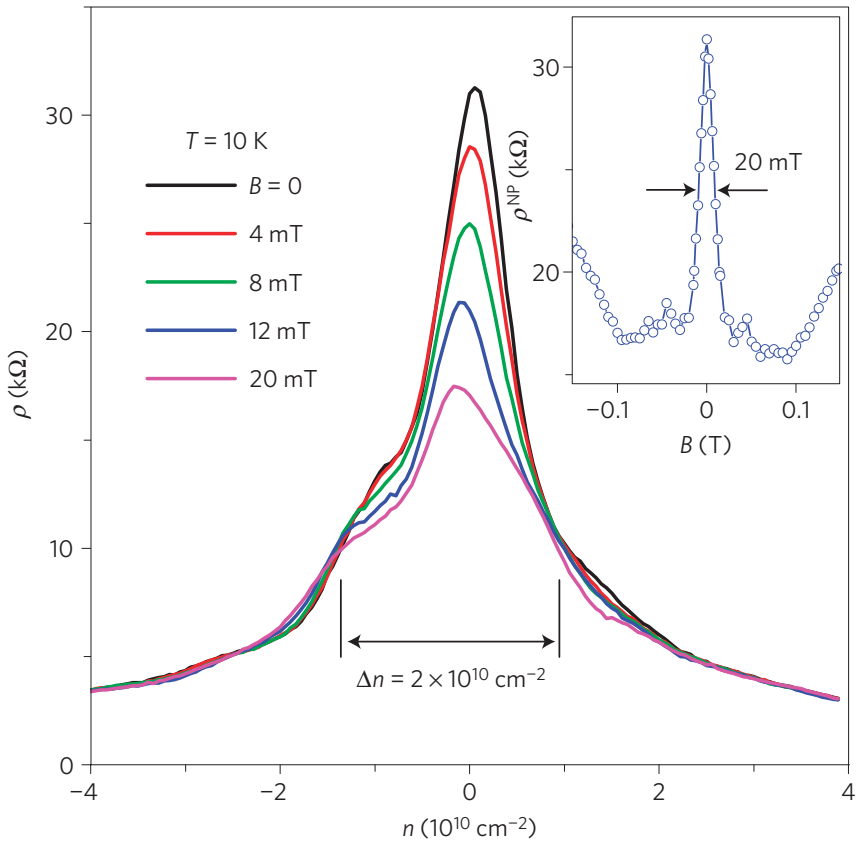

Figure 3 | Resistivity of the studied layer in the insulating regime at various $B . d=12 \mathrm{~nm} ; n_{C} \approx 3 \times 10^{11} \mathrm{~cm}^{-2}$. At low $T, \rho(n)$ exhibits pronounced 'mesoscopic' fluctuations (for example, the left shoulder in this figure) which develop further with decreasing $T$ and are probably due to macroscopic charge inhomogeneity. Inset-detailed $B$ dependence of $\rho^{\mathrm{NP}}$ for the case shown in the main figure.

this contradicts the fact that $\mu$ can increase notably at high $n_{\mathrm{C}}$, that is, graphene exhibits higher quality rather than extra scattering if the control layer is strongly doped (Supplementary Information). Moreover, the Coulomb interaction between the layers is generally expected to become less efficient with decreasing $T$ and increasing $n_{\mathrm{C}}$ (ref. 27), which is exactly opposite to what we observe. Finally, an interlayer scattering mechanism can be ruled out by the fact that any interaction potential created by carriers in the control layer and acting on the studied layer varies at distances of $\sim d \gg a(a$ is the lattice constant), whereas the fast components needed for intervalley scattering depend exponentially on $a / d$ (ref. 28).

To explain the MIT, we assume a small amount of intervalley scatterers already present in our devices. They could be either some of the defects that limit $\mu$ (for example, strong adsorbates) ${ }^{29}$ or, alternatively, the intervalley scattering can arise because of the atomic-scale potential created by BN. In both cases, this can break down the symmetry between the carbon sublattices and act as a source of intervalley scattering. Because the insulating state is observed only for $|n| \leq 10^{10} \mathrm{~cm}^{-2}$ and the process responsible for Anderson localization should provide a mean free path of about $\lambda_{\mathrm{F}}=(4 \pi / n)^{1 / 2}$, we can estimate the intervalley scattering length $l_{\text {iv }}$ as $\sim 0.1 \mu \mathrm{m}$.

Furthermore, $B^{*} \sim 10 \mathrm{mT}$ yields a spatial scale $\left(\phi_{0} / B^{*}\right)^{1 / 2} \approx$ $0.5 \mu \mathrm{m}$, which corresponds to a flux quantum $\phi_{0}=h / e$ enclosed by diffusive trajectories. This scale is significantly larger than the mean free path $l \leq 0.1 \mu \mathrm{m}$ estimated for the relevant interval of $n \leq 10^{10} \mathrm{~cm}^{-2}$ and, therefore, this justifies the use of diffusive transport concepts. Fitting the magnetoresistance curves, such as in Fig. 3, by the weak localization formulas ${ }^{1,30}$ (although mentioning that those are applicable to small rather than large changes in $\rho$ ) yields two other spatial scales. One corresponds to the onset of magnetoresistance $(\sim 1 \mathrm{mT})$ and yields the phase-breaking length of a few $\mu \mathrm{m}$ at liquid-helium $T$, which is typical for graphene $e^{30,31}$. The other scale $(\approx 0.1 \mu \mathrm{m})$ is given by $B \approx 0.1 \mathrm{~T}$, where the magnetoresistance saturates, before changing its sign from negative to positive. The latter scale could be due to the onset of intervalley scattering ${ }^{18,19,31}$, which agrees well with the value $l_{\text {iv }}$ determined from the above analysis of the MIT.

The proposed scenario for the MIT can be considered routine for any high- $\rho$ metallic system at low $T$, including the previously studied damaged graphene, which contains a large amount of short-range, intervalley scatterers ${ }^{7-10}$. Quality graphene has been the only known exception until now. Therefore, the question should be turned around and it should be asked why there is no MIT in the standard graphene devices or DLG at low $n_{\mathrm{C}}$ and why the MIT becomes pronounced only in our ultra-high-quality graphene. The latter seemingly contradicts the very notion of Anderson localization. The puzzle has a straightforward resolution if we attribute this behaviour to the presence of electron-hole puddles ${ }^{4,23,24}$.

In graphene on $\mathrm{SiO}_{2}$, the puddles contain carriers in typical $n \sim 10^{11} \mathrm{~cm}^{-2}$ (ref. 20). In GBN, puddles are larger and shallower ${ }^{21,22}$ but, within each puddle, $n$ is still high enough $\left(>10^{10} \mathrm{~cm}^{-2}\right)$ to move the system away from the MIT. The resistivity of such an inhomogeneous system is then determined by inter-puddle ballistic transport with $\rho \sim h / 4 e^{2}$ (refs 23,24). The recovery of the MIT can be expected if $n$ within the electron-hole puddles decreases below the localization threshold $\left(\approx 10^{10} \mathrm{~cm}^{-2}\right.$ in our case). Accordingly, we attribute the influence of the control layer to the fact that at high $n_{\mathrm{C}}$ it screens out the background potential, making puddles shallower, as our numerical modelling shows (see Supplementary Information). Experimentally, this is also the case, as seen from Hall measurements where the transition region in $\rho_{x y}$ (between electron- and hole-regimes) narrows at high $n_{\mathrm{C}}$ (Supplementary Information). Further work is required to understand the underlying physics in detail and, especially, the mechanism of intervalley scattering and a possible role of the atomic washboard created by BN.

Received 23 June 2011; accepted 9 September 2011; published online 9 October 2011

\section{References}

1. Kramer, B. \& Mackinnon, A. Localization-theory and experiment. Rep. Prog. Phys. 56, 1469-1564 (1993).

2. Imada, M., Fujimori, A. \& Tokura, Y. Metal-insulator transitions. Rev. Mod. Phys. 70, 1039-1263 (1998).

3. Evers, F. \& Mirlin, A. D. Anderson transitions. Rev. Mod. Phys. 80, 1355-1417 (2008).

4. Geim, A. K. \& Novoselov, K. S. The rise of graphene. Nature Mater. 6, 183-191 (2007).

5. Castro Neto, A. H. et al. The electronic properties of graphene. Rev. Mod. Phys. 81, 109-162 (2009).

6. Gomez-Navarro, C. et al. Electronic transport properties of individual chemically reduced graphene oxide sheets. Nano Lett. 7, 3499-3503 (2007).

7. Zhou, S. Y., Siegel, D. A., Fedorov, A. V. \& Lanzara, A. Metal to insulator transition in epitaxial graphene induced by molecular doping. Phys. Rev. Lett. 101, 086402 (2008).

8. Bostwick, A. et al. Quasiparticle transformation during a metal-insulator transition in graphene. Phys. Rev. Lett. 103, 056404 (2009).

9. Elias, D. C. et al. Control of graphene's properties by reversible hydrogenation: Evidence for graphane. Science 323, 610-613 (2009).

10. Chen, J. H. et al. Defect scattering in graphene. Phys. Rev. Lett. 102, 236805 (2009).

11. Adam, S. \& Das Sarma, S. Boltzmann transport and residual conductivity in bilayer graphene. Phys. Rev. B 77, 115436 (2008).

12. Nersesyan, A. A., Tsvelik, A. M. \& Wenger, F. Disorder effects in 2-dimensional d-wave superconductors. Phys. Rev. Lett. 72, 2628-2631 (1994).

13. Ludwig, A. W. W., Fisher, M. P. A., Shankar, R. \& Grinstein, G. Integer quantum Hall transition-an alternative approach and exact results. Phys. Rev. B 50, 7526-7552 (1994).

14. Hatsugai, Y., Wen, X. G. \& Kohmoto, M. Disordered critical wave functions in random-bond models in two dimensions: Random-lattice fermions at $E=0$ without doubling. Phys. Rev. B 56, 1061-1064 (1997).

15. Ryu, S. \& Hatsugai, Y. Singular density of states of disordered Dirac fermions in chiral models. Phys. Rev. B 65, 033301 (2002).

16. Katsnelson, M. I., Novoselov, K. S. \& Geim, A. K. Chiral tunnelling and the Klein paradox in graphene. Nature Phys. 2, 620-625 (2006). 
17. Cheianov, V. V. \& Fal'ko, V. I. Selective transmission of Dirac electrons and ballistic magnetoresistance of $\mathrm{n}-\mathrm{p}$ junctions in graphene. Phys. Rev. B 74, 041403 (2006).

18. Aleiner, I. L. \& Efetov, K. B. Effect of disorder on transport in graphene. Phys. Rev. Lett. 97, 236801 (2006).

19. McCann, E. et al. Weak-localization magnetoresistance and valley symmetry in graphene. Phys. Rev. Lett. 97, 146805 (2006).

20. Martin, J. et al. Observation of electron-hole puddles in graphene using a scanning single-electron transistor. Nature Phys. 4, 144-148 (2008).

21. Xue, J. M. et al. Scanning tunnelling microscopy and spectroscopy of ultra-flat graphene on hexagonal boron nitride. Nature Mater. 10, 282-285 (2011).

22. Decker, R. et al. Local electronic properties of graphene on a BN substrate via scanning tunnelling microscopy. Nano Lett. 11, 2291-2295 (2011).

23. Adam, S., Hwang, E. H., Galitski, V. M. \& Das Sarma, S. A self-consistent theory for graphene transport. Proc. Natl Acad. Sci. USA 104, 18392-18397 (2007).

24. Cheianov, V. V., Fal'ko, V. I., Altshuler, B. L. \& Aleiner, I. L. Random resistor network model of minimal conductivity in graphene. Phys. Rev. Lett. 99, 176801 (2007).

25. Dean, C. R. et al. Boron nitride substrates for high-quality graphene electronics. Nature Nanotech. 5, 722-726 (2010).

26. Mayorov, A. S. et al. Micrometer-scale ballistic transport in encapsulated graphene at room temperature. Nano Lett. 11, 2396-2399 (2011).

27. Tse, W. K., Hu, B. Y. K. \& Das Sarma, S. Theory of Coulomb drag in graphene. Phys. Rev. B 76, 081401 (2007).

28. Ponomarenko, L. A. et al. Effect of a high- $\kappa$ environment on charge carrier mobility in graphene. Phys. Rev. Lett. 102, 206603 (2009).
29. Ni, Z. H. et al. On resonant scatterers as a factor limiting carrier mobility in graphene. Nano Lett. 10, 3868-3872 (2010).

30. Tikhonenko, F. V., Horsell, D. W., Gorbachev, R. V. \& Savchenko, A. K. Weak localization in graphene flakes. Phys. Rev. Lett. 100, 056802 (2008).

31. Tikhonenko, F. V., Kozikov, A. A., Savchenko, A. K. \& Gorbachev, R. V. Transition between electron localization and antilocalization in graphene. Phys. Rev. Lett. 103, 226801 (2009).

\section{Acknowledgements}

We thank M. Katsnelson, A. MacDonald and A. C. Neto for useful discussions. This work was supported by the Engineering and Physical Sciences Research Council (UK), the Royal Society, the Office of Naval Research, the Air Force Office of Scientific Research and the Körber Foundation.

\section{Author contributions}

R.V.G. designed the experiments; L.A.P. and S.V.M. carried out measurements; R.V.G. and R.J. fabricated the devices; L.A.P., R.V.G. and A.K.G. carried out data analysis; L.A.P., A.A.Z. and A.K.G. wrote the article. All the authors contributed to discussions. L.A.P. and R.V.G. contributed to the work equally.

\section{Additional information}

The authors declare no competing financial interests. Supplementary information accompanies this paper on www.nature.com/naturephysics. Reprints and permissions information is available online at http://www.nature.com/reprints. Correspondence and requests for materials should be addressed to R.V.G. 